\title{
DoE-based performance optimization of energy management in sensor nodes powered by tunable energy-harvesters
}

\author{
Tom J Kazmierski, Leran Wang, Bashir Al-Hashimi, Geoff Merrett \\ Faculty of Physics and Applied Science, University of Southampton \\ [tjk|lw04r|bmah|gvm]@ecs.soton.ac.uk
}

\begin{abstract}
An energy-harvester-powered wireless sensor node is a complicated system with many design parameters. To investigate the various trade-offs among these parameters, it is desirable to explore the multi-dimensional design space quickly. However, due to the large number of parameters and costly simulation CPU times, it is often difficult or even impossible to explore the design space via simulation. A design of experiment (DoE) approach using the response surface model (RSM) technique can enable fast design space exploration of a complete wireless sensor node powered by a tunable energy harvester. As a proof of concept, a software toolkit has been developed which implements the DoE-based design flow and incorporates the energy harvester, tuning controller and wireless sensor node. Several test scenarios are considered, which illustrate how the proposed approach permits the designer to adjust a wide range of system parameters and evaluate the effect almost instantly but still with high accuracy..
\end{abstract}

Keywords-energy harvesters, design of experiment, wireless sensor nodes

\section{INTRODUCTION}

Kinetic energy harvesters can be implemented to power wireless sensors thereby eliminating the need for batteries. Such systems are extremely complex, as they operate in multiple technology domains: analogue electrical, digital, mechanical and magnetic. Energy harvesters, specifically those with mechanical resonant frequency tuning, which can match the dominant input frequency, are deemed to be a suitable power source for wireless sensor nodes[1]. Energy consumption optimisation and other energy-related performance issues in wireless sensor networks (WSNs) are becoming more and more important, due to many emerging applications such as environmental sensing, structural monitoring and pervasive healthcare. Design of such systems is a difficult problem due to the need to optimize the entire sensor node holistically[2]. The complexity leads to prohibitive simulation times. Models based on hardware description languages (HDL), such as VHDL-AMS and SystemC-A have recently been proposed[2,3] to accelerate simulation times. However, these solutions have not fully solved the main challenge of the traditional analogue simulation approach based on Newton-Raphson iterations which are the main cause of the long CPU times. We have developed a linearized statespace technique which can reduce the CPU time of one simulation by two orders of magnitude[4], but, as many thousands of simulations are required in traditional, simulationbased optimisation, it is still not possible to design efficiently a complete wireless sensor node. Classical multi-variable optimization methods are based on gradient searches and various heuristic algorithms, such as genetic optimization or simulated annealing. Although these techniques allow improvements to an initial design, they are difficult to use, due to long CPU times, to fast design space exploration and fast investigation of possible trade-offs.

\section{DOE-BASED APPROACH}

DoE was first adopted as a formal approach in the early $1920 \mathrm{~s}$ in agricultural, experimental research. Nowadays, although DoE approaches are common practice in industrial and scientific experimentation, they are rarely taken up by the Electronic Design Automation community to tackle serious problems. We have recently carried out research focused on combining DoE with HDL-based modelling of a complex energy-harvesting sensor node to enable designers to explore the available design space very quickly, without the need for costly simulations. A moderate number of simulations is required initially to build the RSMs, but once the design space is approximated and captured, its exploration is very fast. A number of sensor node performance indicators can be evaluated very quickly as functions of selected design parameters. A moderate number of simulations are required to built the RSM but afterwards exploration of the design space and investigation of trade-offs between the design parameters and performance indicators is practically instant.

\section{REFERENCES}

[1] S. Roundy, P. K. Wright, and J. M. Rabaey, Energy scavenging for wireless sensor networks: with special focus on vibrations. Springer, 2004.

[2] T.J. Kazmierski, G. Merrett, L.Wang, B. Al-Hashimi, A.Weddell,and I. Ayala Garcia, "Modeling of wireless sensor nodes powered by tunable energy harvesters: Hdl-based approach," IEEE Sensors Journal, vol. 12, no. 8, pp. 2680-2689, 2012.

[3] H. Boussetta, M. Marzencki, S. Basrour, and A. Soudani, "Efficient physical modeling of MEMS energy harvesting devices with VHDLAMS,” IEEE Sensors Journal, vol. 10, no. 9, pp. 1427-1437, 2010.

[4] T. J Kazmierski, L. Wang, B. Al-Hashimi, and G. Merrett, "An explicit linearized state-space technique for accelerated simulation of electromagnetic vibration energy harvesters," IEEE Transactions on Computer-Aided Design of Integrated Circuits and Systems, vol. 31, no. 4, pp. 522-531, 2012.

978-3-9815370-0-0/DATE13/@2013 EDAA 